\title{
New Developments in the Management of Laryngeal Cancer
}

\author{
${ }^{1}$ Omar Mirza, ${ }^{2}$ Sean Loughran
}

\begin{abstract}
Laryngeal cancer remains one of the most common airway cancers. Although the incidence has generally declined, the overall survival has actually decreased over the last 40 years despite advancements in its treatment. Treating a malignancy in this region is challenging, and management involves a balance between primary oncological control, organ and functional preservation, and minimizing treatment morbidity. Patients with laryngeal cancer require a truly multidisciplinary team approach. Surgery remains an integral part of management, with several viable organ preservation-based surgical approaches evolving over recent years. There have also been significant advancements in other nonsurgical laryngeal preservation treatment modalities, such as radiation and chemotherapy. However, there still remains a need for further research in understanding the disease, and more innovation in its treatment. Future research in the treatment of laryngeal cancer should be focused on strategies to improve locoregional control and overall survival, while reducing patient morbidity and the impact on quality of life.
\end{abstract}

Keywords: Chemotherapy, Intensity-modulated radiotherapy, Laryngeal cancer, Larynx transplant, Organ preservation, Proton beam, Quality of life, Radiotherapy, Robotic surgery, Total laryngectomy, Transoral surgery.

How to cite this article: Mirza O, Loughran S. New Developments in the Management of Laryngeal Cancer. Int J Head Neck Surg 2018;9(2):54-60.

Source of support: Nil

Conflict of interest: None

\section{INTRODUCTION}

Laryngeal cancer is the second most common airway cancer after lung cancer and has an estimated global incidence of 157000 per annum, making it the sixth most common cancer worldwide. ${ }^{1,2}$ The histopathology of laryngeal cancer consists almost entirely of squamous cell

\footnotetext{
${ }^{1}$ Specialty Registrar, ${ }^{2}$ Consultant Otolaryngologist, Head, Neck and Thyroid Surgeon

1,2Department of ENT, Head and Neck Surgery, Manchester Royal Infirmary, Manchester University NHS Foundation Trust Manchester, UK

Corresponding Author: Sean Loughran, Consultant Otolaryngologist, Head, Neck and Thyroid Surgeon, Department of ENT, Head and Neck Surgery, Manchester Royal Infirmary, Manchester University NHS Foundation Trust, Manchester, UK, Phone: +01619019257, e-mail: Sean.loughran @mft.nhs.uk
}

carcinoma (SCC) in up to $95 \%$ of cases. There are gender and racial disparities with laryngeal cancer occurring almost five times more commonly in men than in women, and with the African-American population presenting at a younger age, with a higher incidence and with greater mortality compared with Caucasians. ${ }^{3-5}$ Several risk factors have been identified in the pathogenesis of laryngeal cancer, the most significant being tobacco and alcohol consumption, which have both been shown to have a risk proportional to the intensity and duration of consumption, and in combination to have a multiplicative effect on risk. ${ }^{6,7}$ The oncogenic role of human papillomavirus (HPV) infection, specifically subtypes 16 and 18 , is also recognized in the development of laryngeal cancer. It is estimated that the prevalence of HPV ranges between $20 \%$ and $30 \%$ in laryngeal cancer; however, its biologic role in the development of malignancy and prognostic relevance remains unclear. ${ }^{8,9}$

The incidence of laryngeal cancer has generally declined over the last 40 years largely due to the reduction in rates of smoking. ${ }^{10}$ Historically, surgery has been the mainstay and still remains an integral part of treatment, but other nonsurgical treatment modalities, such as radiation and chemotherapy have developed as viable organ-preserving options. The unique anatomy of the larynx is complex and contains structures responsible for vital functions, such as breathing, airway protection, and speaking. Treating a malignancy in this region is therefore, challenging, and management involves a balance between primary oncological control and aims to preserve and, where possible, restore form and function, while minimizing treatment morbidity. Nevertheless, unique morbidities are observed with this disease and the care of these patients truly requires a multidisciplinary teambased approach. Although there have been significant advancements in treatment, unfortunately, the overall 5 -year survival rate has actually decreased over the past 40 years, perhaps highlighting the need for further research in understanding the disease, and more innovation in its treatment. ${ }^{11,12}$ In this article, we review current treatment concepts, and discuss new and potential future developments in the management of laryngeal cancer.

\section{SURGERY}

The principles of oncologic surgery in the larynx have evolved as our understanding of the pathways of tumor 
spread has developed and improved surgical techniques have become available. Early-stage laryngeal tumors were traditionally treated with radiotherapy (XRT), but there is now considerable evidence that supports the use of organ preservation-based surgical approaches. ${ }^{13}$ These may involve transoral surgery, which may be microscopic or endoscopic, laser or robotic, or open laryngeal procedures, such as supraglottic, supracricoid, vertical or partial laryngectomy. Conservation surgery relies on the principles of the preservation of the cricoid cartilage, which is necessary to maintain integrity of the airway, and conservation of a cricoarytenoid complex (consisting of a single arytenoid, intact cricoid, full complement of muscles, and recurrent laryngeal nerve innervation), essential to maintain a safe airway. ${ }^{14}$ Early-stage localized tumors in carefully selected patients are more amenable to organ preservation surgery and have equal, if not more, favorable locoregional disease control, overall survival, and functional outcomes.

\section{Transoral Laser Microsurgery}

Transoral laser microsurgery (TLM) has become an established surgical treatment modality for early-stage glottic and supraglottic tumors (Tis, T1a, T1b, and T2). ${ }^{12}$ It has also increasingly been used for more radical resections of advanced tumors. It offers precise cutting and coagulation with a $\mathrm{CO}_{2}$-laser and enhanced microscopic exposure permitting excellent oncological control, low morbidity rate, and good functional outcomes. Two-millimeter surgical margins have been shown to be adequate for transoral excision of tumors; however, it may be difficult to achieve safe margins with excision of tumors in certain anatomical areas, such as the anterior commissure, which remains best treated with XRT, but may still be associated with worse outcomes compared with other early-stage laryngeal tumors. ${ }^{15}$ Local control rate and overall survival have been shown to be equivalent to XRT alone, but with the added advantage that further surgery or XRT could both remain treatment options in the future should there be recurrence. ${ }^{14,16}$ Voice recovery and outcomes have also been shown to be comparable to those after XRT. ${ }^{17,18} \mathrm{~A}$ further advantage of TLM is that excision will usually involve a single procedure, whereas XRT would involve multiple treatments over several weeks.

\section{Transoral/Open Partial Laryngectomy}

Several variations of organ preservation surgical techniques exist. Open conservation procedures, such as supraglottic and supracricoid laryngectomy are increasingly less indicated, especially with greater advances in performing this type of surgery via a transoral approach, which, although technically more challenging, carries with it less morbidity and greater cost efficiency. An open approach may, however, be appropriate in patients who wish to avoid XRT, but may not be candidates for a transoral approach. However, patients with significant pulmonary or systemic comorbidities, or who are at high risk for pulmonary aspiration may not be suitable. Tumors which extend to the subglottis involve the posterior commissure, have through-and-through cartilage involvement, or involve a fixed cricoarytenoid complex would also be unsuitable for partial or supracricoid laryngectomy. ${ }^{13}$ Locoregional control and overall survival outcomes after supraglottic and supracricoid laryngectomy, however, have been shown to be comparative to patients treated with XRT for early-stage tumors. ${ }^{19}$ Intensive rehabilitation is often required to aid restoration of swallowing and speech, with voice outcomes, although substantially different postoperatively, thought to be equivalent to patients treated with XRT. Partial laryngectomy techniques could also be considered in the salvage setting when XRT or chemotherapy has failed. In comparison with patients with total laryngectomy, quality of life, function, and voice outcomes have been shown to be far greater, although the subset of patients in which this type of surgical resection is possible is relatively small. ${ }^{20}$

\section{Transoral Robotic Surgery}

Transoral robotic surgery (TORS) has become increasingly popular over the last two decades and is gaining increasing importance in head and neck surgical oncology. The advantages of robotic surgery are a wider three-dimensional surgical field through smaller surgical access, allowing very precise, tremor-free movement in spaces that may not be accessible using nonrobotic instruments. It provides an alternate approach to open surgery and conventional transoral techniques. Although there is a lack of published data on the application of TORS in laryngeal cancer, preliminary individual surgeon and institutional experiences on the feasibility and adequacy of this surgical technique have been promising. ${ }^{21,22}$ However, the role of TORS in the surgical management of laryngeal cancer needs to be further defined, and the long-term oncological and functional outcomes investigated.

\section{Management of the Neck}

Treatment of the neck in laryngeal cancer has not changed much in recent years, and still depends on the laryngeal subsite and knowledge of its lymphatic drainage. Patients with nodal metastasis $(\mathrm{N}+)$ require definitive treatment either by a comprehensive neck dissection or definitive 
chemoradiotherapy (CRT). As a general rule, elective treatment of a N0 neck is only necessary if the risk of developing occult metastasis is greater than $20 \%$. Tumors in each laryngeal subsite have different clinical behaviors. The supraglottis has a rich bilateral lymphatic drainage; therefore, relatively early-stage disease (T2 or greater) would carry with it risk of bilateral nodal metastasis and would require bilateral treatment of the neck. The glottis, in comparison, has poor lymphatic drainage and would only require unilateral management of the neck for tumors T3 or greater.

\section{Salvage Surgery}

Patients who fail nonsurgical organ preservation techniques require salvage surgery in the form of a total laryngectomy, which serves as the only viable treatment option with curative intent. Local recurrence of the primary tumor occurs in approximately 30\% of patients with advanced-stage laryngeal cancer submitted to nonsurgical organ-preserving treatments. ${ }^{23}$ Rates of complications after salvage surgery tend to be higher, thought to be as a result of impaired wound healing secondary to poor vascular supply following XRT or CRT. The rate of pharyngocutaneous fistula can be as high as 30\%, and fistula formation can result in significant morbidity with a delay in oral intake and extended inpatient stay. ${ }^{24}$ A local pedicled flap could be used as an overlay if primary pharyngeal closure is achieved, but there are concerns regarding tissue vitality to both reinforce the closure and deliver an enhanced vascular supply. Pharyngeal reconstruction may be necessary and can be achieved through several reconstructive options, each with its own advantages and disadvantages. Multi-institutional studies have demonstrated a significant reduction in fistula rates with local pedicled and free flaps, and a reduction in the duration of a fistula in the presence of free tissue graft. ${ }^{25}$ Controversy exists with regard to the elective management of the neck for N0 disease in patients undergoing salvage laryngectomy. There are variable data and treatment philosophies in the literature; however, on balance, it would be reasonable to consider elective neck dissection for locally advanced tumors. Two-year overall survival rates following salvage surgery have been shown to be up to $76 \%$, and 5-year disease-specific survival in earlystage disease up to $70 \%$, and in advanced-stage cancer, $55 \% .{ }^{24,26}$ Functional outcomes, especially swallowing may be poor, but vocal rehabilitation has advanced over recent years with the use of tracheoesophageal prosthesis, which is considered superior to esophageal and electrolarynx speech. Preoperative counseling and postprocedure intensive speech and swallow therapy is essential to avoid the psychosocial implications of this type of surgery.

\section{Tissue-engineered Larynx}

The limitations of current treatment modalities have led some to explore the idea of tissue engineering as a therapeutic option. This involves the application of biological and material science to engineer a synthetic replacement to a nonfunctional organ. The transplant of a cadaveric larynx has been described in the literature, however, with poor long-term outcomes. ${ }^{27}$ The concept of transplanting a biocompatible cartilage scaffold covered in the patient's own epithelium, which integrates with the host tissue thereby negating the rejection process, would revolutionize the treatment of laryngeal cancer and improve the prospect of organ and functional preservation. ${ }^{28}$ There are multiple challenges which exist, however, before this concept can be transferred into clinical practice, and this may take at least another 10 years to see fruition.

\section{RADIOTHERAPY}

The XRT plays an integral role in curative-intent therapy of laryngeal cancer, either as a definitive nonsurgical organ preservation treatment or as an adjuvant therapy in the postoperative period. The XRT techniques, field, dose, and fractionation vary depending on the primary site and extent of the laryngeal cancer and its spread to the neck. The primary sites of laryngeal cancer are either supraglottic or glottic, while disease in the subglottis is generally considered to be tumor extension rather than a primary cancer in its own right, which, although possible, is rare. The main aim of XRT is to achieve local control while minimizing damage to surrounding normal tissues and structures, which can present a treatment challenge paradigm owing to the close proximity of critical organs in an anatomically complex region. Early and late radiation-related morbidity, including dysphagia, xerostomia, and mucositis, among others, can have a significant impact on quality of life and functional outcomes, which certainly require thought when managing patients with laryngeal cancer.

Early-stage glottic primaries can be treated effectively with either single-modality XRT or surgery with similar excellent outcomes. Five-year control rates for T1 glottic tumors treated with definitive XRT have been reported up to $93 \%$, and T2 tumors up to $73 \% .{ }^{29,30}$ As the glottis has fairly poor lymphatic drainage, early disease in this region rarely spreads through adjacent lymphatics; therefore, elective treatment of the neck is often unnecessary. For T1 and T2 lesions, XRT is generally delivered via opposed lateral fields comprising the thyroid cartilage superiorly, the cricoid cartilage, or first tracheal ring 
inferiorly, with the vertebra forming the posterior field limit. The combination of this method and the anterior contour of the neck may result in underdosing of the anterior commissure; therefore, an augmented dose should be considered for disease in this region owing to its greater risk of recurrence and association with worse local control compared with other T1 tumors. ${ }^{31}$ A higher dose per fraction has been shown to give improved local control, with the generally accepted total dose for $\mathrm{T} 1$ lesions being $63 \mathrm{~Gy}$, and for T2 lesions, $65 \mathrm{~Gy}{ }^{31,32} \mathrm{~A}$ disadvantage of XRT worth considering is the degree of posttreatment tissue edema which may impair both visual and histological examination during subsequent surveillance and potentially result in a delay in diagnosis of recurrent disease, increasing the chance that surgical salvage would necessitate total laryngectomy. ${ }^{33,34}$

Early-stage supraglottic tumors can also be effectively treated with definitive XRT, with local control rates for $\mathrm{T} 1$ tumors ranging between $73 \%$ and $100 \%$ and for T2 tumors, between $60 \%$ and $89 \%{ }^{35}$ The supraglottis, in contrast to the glottis, has a rich lymphatic drainage, and even early-stage supraglottic disease may present with nodal metastasis or harbor subclinical nodal disease requiring elective management with XRT at the same sitting to bilateral neck levels II through IV. The standard radiation treatment volumes for early-stage supraglottic cancer is a total dose of 70 Gy to the primary site, and 50 Gy to bilateral neck if lymph node negative or a higher dose of 60 Gy to involved lymph node or high-risk areas. ${ }^{12}$

The XRT has a role in adjuvant therapy after surgery for advanced-stage laryngeal cancer. Pathological risk factors which are taken into account when considering postoperative XRT include multiple positive nodes, perineural invasion, lymphovascular invasion, close resection margins, or soft tissue or thyroid cartilage involvement. The addition of chemotherapy, if there are positive resection margins and extracapsular nodal extension, has been shown to benefit locoregional control and improve overall survival. ${ }^{36}$

\section{Intensity-modulated Radiation Therapy}

Radiation oncology in the head and neck has evolved considerably over recent years with the advent of intensity-modulated XRT (IMRT) and volumetric arc therapy (VMAT), now favored over traditional threeand two-dimensional techniques, which are able to offer precise radiation delivery, while minimizing the dose to surrounding normal structures without compromise of target coverage. ${ }^{37}$ Furthermore, image-guided planning and specific patient immobilization and positioning techniques with the use of a thermoplastic mask are able to expose the larynx and minimize dose delivery to surrounding tissues, while creating a reproducible set-up for the whole duration of XRT treatment. These new techniques avoid exposure to salivary glands, upper aerodigestive mucosa, and pharyngeal constrictors and several studies have reported reduced radiation-related side effects and improved functional and quality-of-life outcomes. ${ }^{38-40}$ Through reducing the burden of normal tissue toxicity, a higher dose of radiation is acceptably delivered to the target, hence improving XRT effectiveness and improved locoregional control.

\section{Fractionation}

The XRT alone is a viable option not only for early-stage glottic and supraglottic disease, but also in patients with advanced disease who are not candidates for surgery or CRT. In this setting, customization of techniques, such as altered dose or fractionation schemes could be considered. Conventional fractionation consists of daily fractions of up to $2 \mathrm{~Gy}$. The main alternate regimes which have been suggested include hyperfractionation and accelerated fractionation. Hyperfractionation involves two or more smaller doses of radiation on each treatment day, thereby allowing a higher biologically effective dose to be delivered to the tumor. Accelerated fractionation refers to the reduced overall treatment time over which the total radiation dose is administered. The rationale for this is that the accelerated dose administration is able to reduce the time available for tumor cell regeneration. Both of these schedules have been shown to be associated with significantly higher locoregional control and survival rates when compared with standard fractionation schedules, although they are associated with more severe acute radiation side effects, such as mucositis, but no greater incidence of late radiation-related complications. ${ }^{41,42}$ However, although considerable variation in practice among different institutions does exist, there is still no optimal treatment structure decisively described, with this area remaining a source of ongoing debate despite extensive research efforts.

\section{Proton Beam Radiation Therapy}

The future direction of XRT would be the optimization of IMRT and VMAT techniques through dose reduction to normal tissues, dose escalation to tumors, and by clearly defining altered fractionation schemes. Despite these measures, a large proportion of patients may still experience radiation-related morbidity with a significant negative impact on quality of life. An exciting area of development in radiation oncology is the use of proton beam radiation therapy (PBRT) which has been used with success in a few centers around the world. Due to 
the unique inherent physical properties of protons, PBRT has the advantage of delivering precise radiation to the tumor but for a significantly lower normal surrounding tissue dose, thereby improving the therapeutic ratio. This is especially relevant for tumors in the head and neck, where preliminary evidence on the use of PBRT has shown significantly reduced radiation-related morbidity compared with conventional XRT, without sacrificing target coverage. ${ }^{43,44}$ The expectation is that PBRT will be more widely available in the future, although there are greater costs compared with conventional XRT, which will need to be rationalized in order for it to be financially viable within the context of the current UK health economy.

\section{CHEMOTHERAPY}

Total laryngectomy was historically the mainstay treatment for advanced-stage laryngeal tumors and remains associated with significant morbidity including a permanent stoma, loss of voice, swallowing difficulties, as well as the associated psychosocial consequences. The implications of many of these factors on a patient's quality of life prompted several trials in the early 1990s which changed the focus of treatment to nonsurgical organ preservation through using induction or concurrent chemotherapy with radiation. ${ }^{23,45}$ Many of the clinical trials, however, showed little difference in locoregional recurrence or overall survival in patients with advancedstage laryngeal cancer treated with CRT compared with patients who underwent total laryngectomy and adjuvant XRT. The VA Larynx Cancer Study Group trial, however, importantly showed that $66 \%$ of those who received induction chemotherapy were able to preserve their larynx, which from a quality-of-life perspective was a considerable gain for this patient group. ${ }^{23}$ Subsequent clinical trials with concurrent chemotherapy with cisplatin and XRT demonstrated far superior laryngeal preservation $(81 \%)$, when compared with induction chemotherapy regimens or XRT alone, therefore, now being established as the standard of care with this treatment technique. $^{46}$

Despite this paradigm shift and widespread use of nonsurgical treatment protocols, as well as data to support its use, there has continued to be a decrease in survival for patients with laryngeal cancer. This higher rate of long-term mortality and equivocal survival benefit in this group unrelated to the disease and laryngeal preservation compared with other treatment regimens may be as a result of late chemotherapy toxicity. There still remains a lack of prospective trials to support nonsurgical organ preservation treatment, and many of the patient groups involved in previous clinical trials may not be entirely reproducible, especially, for example, patients with T4 disease who were often excluded from these studies. Nevertheless, concurrent CRT remains a viable treatment option for a select group of patients, which includes those with a functional larynx, T3-T4 disease with or without neck disease. However, at present, there is no clear high-quality evidence able to guide clinicians in their selection of patients with advanced-stage laryngeal tumors who may benefit from concurrent CRT. Interestingly, some studies have emerged demonstrating a statistically significant survival benefit in patients with advanced-stage laryngeal cancer (T3 and T4a) who underwent total laryngectomy rather than CRT. ${ }^{47,48}$ Although laryngeal preservation should be considered in patients with locally advanced T3 and T4 disease, upfront surgery in those patients with significant functional deficit of the larynx or those unable or unwilling to undergo CRT may be the only option.

\section{Targeted Therapy}

Greater research is needed to develop newer chemotherapeutic agents and combination of drugs which have greater clinical effectiveness and less toxicity. Targeted therapy focusing on inhibition of molecular targets expressed on cancer cells, such as epidermal growth factor receptor is an exciting new area of chemotherapeutics. Agents, such as cetuximab, a monoclonal antibody, have been shown to be as effective as cisplatin in the treatment of head and neck SCC, but with less toxicity. ${ }^{49}$ However, further prospective studies are required to elucidate its oncological effectiveness as an individual agent or in conjunction with others, before its use becomes more widespread.

The role of molecular biological markers in predicting the behavior of certain tumors, measuring response to treatment, and to identify which patients may be resistant or responsive to treatment is also an area of further interest. If a reliable marker could identify a potential nonresponder or a poor response to treatment, then a more aggressive treatment strategy or earlier surgical salvage could be considered to improve overall survival in this cohort. ${ }^{50,51}$ However, further study is required to assess the reliability of currently identified biological markers.

\section{CONCLUSION}

The management of patients with laryngeal cancer requires a truly multidisciplinary team effort including medical and surgical oncologists, specialist nurses, radiologists, as well as wider healthcare professionals, including speech and language therapists and dieticians. During the past three decades, treatment paradigms in 
the management of laryngeal cancer have evolved. The anatomical complexity of the head and neck presents a treatment challenge due to the close proximity of tumors to surrounding normal tissues and structures. Before considering a treatment strategy, the tumor site, stage and individual patient factors, and treatment preference should to be taken into account. Several surgical and nonsurgical treatment modalities for early-stage laryngeal cancers have developed with excellent outcomes and reduced morbidity. In advanced-stage laryngeal cancers, however, there has been a move away from open surgery to multimodal techniques with the aim of organ preservation. The implications of a total laryngectomy including the psychological and social aspects of losing the ability of voice, and impaired swallow plays a significant role in a patient's decision regarding treatment of their cancer. The CRT has increasingly been offered as an alternative in order to preserve the larynx and improve quality of life. However, there is a disparity between the aim of organ preservation, locoregional control, and overall survival. There still remains a lack of evidence that demonstrates organ preservation techniques in advanced-stage laryngeal cancer improve overall survival. At present, no other treatment technique confers a survival advantage over total laryngectomy with adjuvant therapy. Patients should be fully informed with the advantages and disadvantages of laryngeal preservation options in comparison with treatments which include partial or total laryngectomy.

Although there have been considerable advances in the treatment of laryngeal cancer, there is still a need for further research in understanding the disease, and more innovation in its treatment. Future research in the treatment of laryngeal cancer should be focused on the exploration of strategies to improve locoregional control and overall survival, while reducing patient morbidity and the impact on quality of life.

\section{REFERENCES}

1. Cattaruzza MS, Maisonneuve P, Boyle P. Epidemiology of laryngeal cancer. Eur J Cancer B Oral Oncol 1996 Sep;32B(5): 293-305.

2. Siegel R, Ward E, Brawley O, Jemal A. Cancer statistics, 2011: the impact of eliminating socioeconomic and racial disparities on premature cancer deaths. CA Cancer J Clin 2011 Jul-Aug;61(4):212-236.

3. Goodwin WJ, Thomas GR, Parker DF, Joseph D, Levis S, Franzmann E, et al. Unequal burden of head and neck cancer in the United States. Head Neck 2008 Mar;30(3):358-371.

4. Shin JY, Truong MT. Racial disparities in laryngeal cancer treatment and outcome: a population-based analysis of 24,069 patients. Laryngoscope 2015 Jul;125(7):1667-1674.

5. DeSantis C, Naishadham D, Jemal A. Cancer statistics for African Americans, 2013. CA Cancer J Clin 2013 May;63(3): 151-166.
6. Cavavos LC, Soto-Galindo GA, González JL. Laryngeal cancer update: a review. Ann Otolaryngol Rhinol 2017 Aug;4(6):1184.

7. Bosetti C, Gallus S, Franceschi S, Levi F, Bertuzzi M, Negri E, et al. Cancer of the larynx in non-smoking alcohol drinkers and in non-drinking tobacco smokers. Br J Cancer 2002 Aug;87(5):516-518.

8. Gama RR, Carvalho AL, Longatto Filho A, Scorsato AP, López $\mathrm{RV}$, Rautava J, et al. Detection of human papillomavirus in laryngeal squamous cell carcinoma: systematic review and meta analysis. Laryngoscope 2016 Apr;126(4):885-893.

9. Li X, G Lei, Li H, Gao J, Yang Y, Zhou F, et al. Human papillomavirus infection and laryngeal cancer risk: a systematic review and meta-analysis. J Infect Dis 2013 Feb;207(3):479-488.

10. Sturgis EM, Cinciripini PM. Trends in head and neck cancer incidence in relation to smoking prevalence. Cancer 2007 Oct;110(7):1429-1435.

11. Siegel RL, Miller KD, Jemal A. Cancer statistics, 2016. CA Cancer J Clin 2016 Jan-Feb;66(1):7-30.

12. Steuer CE, El-Deiry M, Parks JR, Higgins KA, Saba NF. An update on larynx cancer. CA Cancer J Clin 2017 Jan;67(1): 31-50.

13. Laccourreye O, Laccourreye H, El-Sawy M, Weinstein GS. Supracricoid partial laryngectomy with cricohyoidoepiglottopexy. In: Weinstein GS, Laccourreye O, Brasnu D, Laccourreye $\mathrm{H}$, editors. Organ preservation surgery for laryngeal cancer. San Diego (CA): Singular Publishing Group; 2000. p. 73.

14. Hartl DM, Brasnu DF. Contemporary surgical management of early glottic cancer. Otolaryngol Clin North Am 2015 Aug;48(4):611-625.

15. Ossoff RH, Sisson GA, Shapshay SM. Endoscopic management of selected early vocal cord carcinoma. Ann Otol Rhinol Laryngol 1985 Nov-Dec;94(6 Pt 1):560-564.

16. Swanson MS, Low G, Sinha UK, Kokot N. Transoral surgery vs intensity modulated radiotherapy for early supraglottic cancer a systematic review. Curr Opin Otolaryngol Head Neck Surg 2017 Apr;25(2):133-141.

17. Cohen SM, Garrett CG, Dupont WD, Ossoff RH, Courey MS. Voice-related quality of life in T1 glottic cancer: irradiation versus endoscopic excision. Ann Otol Rhinol Laryngol 2006 Aug;115(8):581-586.

18. Mendelsohn AH, Matar N, Bachy V, Lawson G, Remacle M. Longitudinal voice outcomes following advanced $\mathrm{CO}_{2}$ laser cordectomy for glottic cancer. J Voice 2015 Nov;29(6):772-775.

19. Sperry SM, Rassekh CH, Laccourreye O, Weinstein GS. Supracricoid partial laryngectomy for primary and recurrent laryngeal cancer. JAMA Otolaryngol Head Neck Surg 2013 Nov;139(11):1226-1235.

20. Sanchez-Cuadrado I, Castro A, Bernaldez R, Del Palacio A, Gavilan J. Oncologic outcomes after supracricoid partial laryngectomy. Otolaryngol Head Neck Surg 2011 Jun;144(6): 910-914.

21. Weinstein GS,O'Malley BW,MagnusonJS,CarrollWR,OlsenKD, Daio L, et al. Transoral robotic surgery: a multicenter study to assess feasibility, safety, and surgical margins. Laryngoscope 2012 Aug;122(8):1701-1707.

22. Park YM, Lee WJ, Lee JG, Lee WS, Choi EC, Chung SM, Kim SH. Transoral robotic surgery (TORS) in laryngeal and hypopharyngeal cancer. J Laparoendosc Adv Surg Tech A 2009 Jun;19(3):361-368.

23. Department of Veterans Affairs Laryngeal Cancer Study Group; Wolf GT, Fisher SG, Hong WK, Hillman R, Spaulding M, 
Laramore GE, Endicott JW, McClatchey K, Henderson WG. Induction chemotherapy plus radiation compared with surgery plus radiation in patients with advanced laryngeal cancer. N Engl J Med 1991 Jun 13;324(24):1685-1690.

24. Weber RS, Berkey BA, Forastiere A, Cooper J, Maor M, Goepfert $\mathrm{H}$, et al. Outcome of salvage total laryngectomy following organ preservation therapy: the Radiation Therapy Oncology Group trial 91-11. Arch Otolaryngol Head Neck Surg 2003 Jan;129(1):44-49.

25. Patel UA, Moore BA, Wax M, Rosenthal E, Sweeny L, Militsakh $\mathrm{ON}$, et al. Impact of pharyngeal closure technique on fistula after salvage laryngectomy. JAMA Otolaryngol Head Neck Surg 2013 Nov;139(11):1156-1162.

26. Sandulache VC, Vandelaar LJ, Skinner HD, Cata J, Hutcheson K, Fuller CD, et al. Salvage total laryngectomy after externalbeam radiotherapy: a 20-year experience. Head Neck 2016 Apr;38(Suppl 1):E1962-E1968.

27. Strome M, Stein J, Esclamado R, Hicks D, Lorenz RR, Braun W, et al. Laryngeal transplantation and 40-month follow-up. N Engl J Med 2001 May;344(22):1676-1679.

28. Hamilton NJ, Birchall MA. Tissue-engineered larynx: future applications in laryngeal cancer. Curr Otorhinolaryngol Rep 2017 Mar;5(1):42-48.

29. Mendenhall WM, Amdur RJ, Morris CG, Hinerman RW. T1T2N0 squamous cell carcinoma of the glottic larynx treated with radiation therapy. J Clin Oncol 2001 Oct;19(20):4029-4036.

30. Fein DA, Mendenhall WM, Parsons JT, Million RR. T1-T2 squamous cell carcinoma of the glottic larynx treated with radiotherapy: a multivariate analysis of variables potentially influencing local control. Int J Radiat Oncol Biol Phys 1993 Mar;25(4):605-611.

31. Le QT, Fu KK, Kroll S, Ryu JK, Quivey JM, Meyler TS, et al. Influence of fraction size, total dose, and overall time on local control of T1-T2 glottic carcinoma. Int J Radiat Oncol Biol Phys 1997 Aug;39(1):115-126.

32. Yamazaki H, Nishiyama K, Tanaka E, Koizumi M, Chatani M. Radiotherapy for early glottic carcinoma (T1N0M0): results of prospective randomized study of radiation fraction size and overall treatment time. Int J Radiat Oncol Biol Phys 2006 Jan;64(1):77-82.

33. Steiner W, Vogt P, Ambrosch P, Kron M. Transoral carbon dioxide laser microsurgery for recurrent glottic carcinoma after radiotherapy. Head Neck 2004 Jun;26(6):477-484.

34. Jenckel F, Knecht R. State of the art in the treatment of laryngeal cancer. Anticancer Res 2013 Nov;33(11):4701-4710.

35. Hinerman RW, Mendenhall WM, Amdur RJ, Stringer SP, Villaret DB, Robbins KT. Carcinoma of the supraglottic larynx: treatment results with radiotherapy alone or with planned neck dissection. Head Neck 2002 May;24(5):456-467.

36. Bernier J, Cooper JS, Pajak TF, van Glabbeke M, Bourhis J, Forastiere A, et al. Defining risk levels in locally advanced head and neck cancers: a comparative analysis of concurrent postoperative radiation plus chemotherapy trials of the EORTC (\#22931) and RTOG (\# 9501). Head Neck 2005 Oct;27(10):843-850.

37. Yeh SA. Radiotherapy for head and neck cancer. Semin Plast Surg 2010 May;24(2):127-136.

38. Nutting CM, Morden JP, Harrington KJ, Urbano TG, Bhide SA, Clark C, et al. Parotid-sparing intensity modulated versus conventional radiotherapy in head and neck cancer (PARSPORT): a phase 3 multicentre randomised controlled trial. Lancet Oncol 2011 Feb;12(2):127-136.
39. Eisbruch A, Marsh LH, Martel MK, Ship JA, Ten Haken R, Pu $\mathrm{AT}$, et al. Comprehensive irradiation of head and neck cancer using conformal multisegmental fields: assessment of target coverage and noninvolved tissue sparing. Int J Radiat Oncol Biol Phys 1998 Jun;41(3):559-568.

40. Feng FY, Kim HM, Lyden TH, Haxer MJ, Feng M, Worden $\mathrm{FP}$, et al. Intensity-modulated radiotherapy of head and neck cancer aiming to reduce dysphagia: early dose-effect relationships for the swallowing structures. Int J Radiat Oncol Biol Phys 2007 Aug;68(5):1289-1298.

41. Bourhis J, Overgaard J, Audry H, Ang KK, Saunders M, Bernier J, et al. Hyperfractionated or accelerated radiotherapy in head and neck cancer: a metaanalysis. Lancet 2006 Sep;368(9538): 843-854.

42. Skladowski K, Maciejewski B, Golen M, Pilecki B, Przeorek W, Tarnawski R. Randomized clinical trial on 7-day continuous accelerated irradiation (CAIR) of head and neck cancerreport on 3-year tumour control and normal tissue toxicity. Radiother Oncol 2000 May;55(2):101-110.

43. Romesser PB, Cahlon O, Scher E, Zhou Y, Berry SL, Rybkin A, et al. Proton beam radiation therapy results in significantly reduced toxicity compared with intensity-modulated radiation therapy for head and neck tumors that require ipsilateral radiation. Radiother Oncol 2016 Feb;118(2):286-292.

44. van de Water T, Bijl HP, Schilstra C, Pijls-Johannesma M, Langendijk JA. The potential benefit of radiotherapy with protons in head and neck cancer with respect to normal tissue sparing: a systematic review of literature. Oncologist 2011 Feb;16(3):366-377.

45. Forastiere AA, Goepfert H, Maor M, Pajak TF, Weber R, Morrison W, et al. Concurrent chemotherapy and radiotherapy for organ preservation in advanced laryngeal cancer. N Engl J Med 2003 Nov;349:2091-2098.

46. Forastiere AA, Zhang Q, Weber RS, Maor MH, Goepfert H, Pajak TF, et al. Long-term results of RTOG 91-11: a comparison of three nonsurgical treatment strategies to preserve the larynx in patients with locally advanced larynx cancer. J Clin Oncol 2013 Mar;31(7):845-852.

47. Dziegielewski PT, O'Connell DA, Klein M, Fung C, Singh P, Alex Mlynarek M, Fung D, Harris JR, Seikaly H. Primary total laryngectomy versus organ preservation for T3/T4a laryngeal cancer: a population-based analysis of survival. J Otolaryngol Head Neck Surg 2012 Apr;41(Suppl 1):S56-S64.

48. Grover S, Swisher-McClure S, Mitra N, Li J, Cohen RB, Ahn PH, et al. Total laryngectomy versus larynx preservation for T4a larynx cancer: patterns of care and survival outcomes. Int J Radiat Oncol Biol Phys 2015 Jul;92(3):594-601.

49. Bonner J, Giralt J, Harari P, Spencer S, Schulten J, Hossain A, et al. Cetuximab and radiotherapy in laryngeal preservation for cancers of the larynx and hypopharynx: a secondary analysis of a randomized clinical trial. JAMA Otolaryngol Head Neck Surg 2016 Sep;142(9):842-849.

50. Bradford CR, Zhu S, Wolf GT, Poore J, Fisher SG, Beals T, et al. Overexpression of $\mathrm{p} 53$ predicts organ preservation using induction chemotherapy and radiation in patients with advanced laryngeal cancer. Department of Veterans Affairs Laryngeal Cancer Study Group. Otolaryngol Head Neck Surg 1995 Oct;113(4):408-412.

51. Corvo R, Giaretti W, Sanguineti G, Geido E, Orecchia R, Guenzi $\mathrm{M}$, et al. In vivo cell kinetics in head and neck squamous cell carcinomas predicts local control and helps guide radiotherapy regimen. J Clin Oncol 1995 Aug;13(8):1843-1850. 